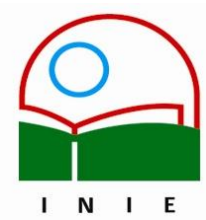

Actualidades Investigativas en Educación

Revista Electrónica publicada por el

Instituto de Investigación en Educación

Universidad de Costa Rica

ISSN 1409-4703

http://revista.inie.ucr.ac.cr

COSTA RICA

\title{
EL CARÁCTER SOCIOCULTURAL DE LA ENSEÑANZA EN LA EDUCACIÓN SUPERIOR
}

THE SOCIOCULTURAL NATURE OF TEACHING IN HIGHER EDUCATION

\author{
Volumen 9, Número Especial
}

pp. 1-23

Este número se publicó el 15 de noviembre 2009

Carmen Obregón Rodríguez

La revista está indexada en los directorios:

LATINDEX, REDALYC, IRESIE, $\underline{\text { CLASE}, ~ D I A L N E T, ~ D O A J, ~ E-R E V I S T @ S, ~}$

La revista está incluida en los sitios:

REDIE, RINACE, OEI, MAESTROTECA, PREAL, HUASCARAN, CLASCO

Los contenidos de este artículo están bajo una licencia Creative Commons 


\title{
EL CARÁCTER SOCIOCULTURAL DE LA ENSEÑANZA EN LA EDUCACIÓN SUPERIOR
}

\author{
THE SOCIOCULTURAL NATURE OF TEACHING IN HIGHER EDUCATION
}

\section{Carmen Obregón Rodríguez ${ }^{1}$}

\begin{abstract}
Resumen: Como la escuela en general, el ámbito de la educación superior también nos ofrece un escenario privilegiado para recabar evidencias del carácter sociocultural del pensamiento. Fundada en las exploraciones de Vigotzky y expandida por sus seguidores, la perspectiva sociocultural establece que nuestro pensamiento en general, y las funciones mentales superiores en particular, están por definición culturalmente mediados; y que no hay forma de no estar culturalmente situados cuando llevamos a cabo una acción, usualmente acorde con los patrones aceptados por el grupo social al que se pertenece (acción mediada). A partir de estas aseveraciones, este ensayo intenta justificar la importancia de un mayor conocimiento del contexto cultural que tiene lugar al interior de la educación superior, y una mejor comprensión de las implicaciones que las diferencias disciplinarias tienen en la enseñanza y el aprendizaje; dar cuenta de algunos hallazgos de incursiones de la investigación empírica a este respecto; y cerrar enmarcando los aportes concretos de este ejercicio dentro de una eminente preocupación por encontrar nuevas formas de articular la multiculturalidad; de integrar la diversidad que nos habita y nos estructura, y que forma parte de nuestras herencias y de nuestro presente cultural.
\end{abstract}

Palabras clave: MEDIACIÓN CULTURAL, INTERDISCIPLINARIEDAD, PRÁCTICAS DOCENTES, EDUCACIÓN SUPERIOR

\begin{abstract}
Just like school does in a general sense, higher education creates a privileged setting for collecting evidences of the sociocultural nature of thinking. Grounded in Vygotsky's explorations and extended by his colleagues, the sociocultural perspective establishes that our thinking in a broad sense, and higher psychological functioning in particular, are by definition culturally mediated; and that there is no other way than to be culturally situated when taking action, usually following patterns accepted by the social group to which one belongs (mediated action). Parting from those statements, this essay attempts to justify the importance of a better understanding of the higher education's cultural context, the implications that disciplinary differences have both for teaching and for learning; also to take into consideration some empirical research findings in the field. All this with a great concern for finding alternatives to promote interdisciplinary dialogue; and ways to understand the diversity that surrounds us and gives us structure; diversity which is part of our natural inheritance and our cultural present.
\end{abstract}

Key words: CULTURAL MEDIATION, INTERDISCIPLINARITY, TEACHING PRACTICES, HIGHER EDUCATION

\footnotetext{
${ }^{1}$ Maestría en Comunicación con especialidad en difusión de la ciencia y la cultura, ITESO. Licenciada en Pedagogía de la Universidad Nacional Autónoma de México, con Mención Honorífica. Actualmente labora para la Universidad Iberoamericana de León, México como Directora del Centro lgnaciano de Formación Humanista. También ha labora en Gestión de servicios educativo universitarios: Área de Reflexión Universitaria (ARU), Fe-Cultura, Pastoral.; programa de maestría en educación humanista; diplomados en filosofía y teología; seminarios, cursos, conferencias. Representante ante el Consejo General Académico y en diversos comités y comisiones.
}

Dirección electrónica: carmen.obregon@leon.uia.mx

Artículo recibido: 26 de mayo, 2009

Aprobado: 19 de octubre, 2009 


\section{Introducción}

Si bien "el enfoque cultural se origina muy estrechamente vinculado al ámbito educativo ya que este permite la adquisición de formas de pensamiento abstracto mediante el uso de instrumentos mediacionales" (Martínez, 2006, p. 29) ${ }^{2}$, en la mayoría de sus aplicaciones en ejercicios de investigación parece haberse centrado en la enseñanza primaria y media, probablemente por la profundidad con que el mismo Vigotsky y sus colaboradores exploraron los orígenes y la evolución de las funciones y procesos psicológicos, y el desarrollo de la relación entre pensamiento y habla (entre otros), en la infancia y la adolescencia.

Sin embargo, el ámbito de la educación superior también ofrece un escenario privilegiado para recabar evidencias del carácter sociocultural del pensamiento $\mathrm{y}$, en particular, de las nociones de funciones mentales superiores, apropiación, conceptos científicos y actividad que no existen fuera de las relaciones sociales que establecemos en nuestro medio, en este caso el educativo.

Dentro del mismo, la docencia constituye una acción de mediación que refleja formas de comportamiento que se organizan y que son definidas de una manera cultural, en función de los patrones aceptados por la disciplina (grupo social) al que se pertenece, las cuales se adquieren a través de la interacción que mantienen sus miembros y se transmiten al cuerpo estudiantil a través de los instrumentos que dicha comunidad disciplinar utiliza para su comunicación.

Utilizando la referencia de Martínez a la escuela, me atrevería a afirmar que la disciplina académica

se caracteriza por propiciar entre sus miembros formas de pensamiento que han sido aceptadas por [ese] grupo cultural, constituyéndose en una de las principales [colectividades] que dota de nuevas formas de enfrentar los problemas a través del desarrollo de artefactos culturales comunes (2006, p. 15), como lo demuestran los experimentos de Galperin (1987) y Davidov (1988).

Por tanto, partiendo de la naturaleza cultural y discursiva de las disciplinas académicas, esta reflexión pretende sostener que el personal docente universitario traduce,

${ }^{2}$ En lugar de citar textualmente a Vigotsky y sus colegas, utilizo como referencia el trabajo de Miguel Angel Martínez por considerar que expresa el enfoque de éstos de manera apropiada para los fines de este ensayo. 
a través de sus prácticas, las representaciones sociales de su disciplina en una racionalidad pedagógica específica que, al ser coincidente con dichas representaciones, las valida y reproduce.

Transmitir los supuestos principales de la disciplina a futuros integrantes de la comunidad, es parte del continuum de comunicación de la ciencia. Implica producir sentido, persuadir de su interés e importancia, formar opiniones favorables, y encauzar en ciertas direcciones los intereses y hasta la conducta de los y las estudiantes.

Resulta entonces importante un mayor conocimiento del contexto cultural que tiene lugar al interior de la educación superior, y una mejor comprensión de las causas e implicaciones que las diferencias disciplinarias tienen en la enseñanza y el aprendizaje; así como dar cuenta de algunas de las incursiones de la investigación empírica en este ámbito.

En lo referente a sus implicaciones para la enseñanza, el conocimiento de las diferencias disciplinares es aún débil, pese a estar en constante desarrollo.

En contraste con un reconocimiento general de que el cuerpo académico se identifica fuertemente con su disciplina, la cuestión de si la enseñanza varía dependiendo del área de conocimiento y cómo es un enfoque relativamente nuevo, que ha recibido poca atención: "Los estudios sobre enseñanza y aprendizaje a nivel universitario permanecen esencialmente enfocados en aspectos genéricos, con lo que bloquean su complejidad y diversidad" (Neumann, 2001, p. 135).

Aún cuando la investigación ha demostrado que mucho del saber sobre la enseñanza específica de una disciplina está implícito y que la cultura disciplinaria es un mediador clave de otras culturas que las estudiantes y los estudiantes encuentran en su experiencia de aprendizaje universitario (Neumann, 2001), el cuerpo investigador ha pasado por alto las creencias educativas de las disciplinas. La variación por disciplina, componente de dichos estudios, raramente es el enfoque preferencial de estas investigaciones (Neumann, 2001).

El conocimiento de la estructura y organización de las disciplinas, de la cultura y el contexto en los que tiene lugar la enseñanza, de las causas y consecuencias que estas diferencias implican en lo educativo, permitiría mejorar la calidad de la instrucción y los resultados de la enseñanza.

La serie de referencias aludidas coinciden en la necesidad de estudios a micronivel que busquen explicaciones a las diferencias sistemáticas entre disciplinas y que puedan ser usados para sustentar políticas institucionales más efectivas; para revisar las prácticas docentes y poder sugerir metodologías que mejoraran el proceso enseñanza-aprendizaje. 
El tema resulta relevante en la apuesta por el diálogo interdisciplinar que exige una educación acorde con los nuevos problemas, mapas laborales y modos de trabajar; como se abordará en las conclusiones del ensayo.

\section{Las culturas disciplinarias}

Con algunas diferencias y variantes menores, considerar las grandes divisiones del conocimiento como "culturas" constituye una apreciación ampliamente difundida.

Si bien C. P. Snow (1963) trae este debate a los terrenos de las disciplinas abordando por primera vez en el campo de la vida académica la separación entre ciencias y cultura literaria, la discusión en terrenos más amplios parece existir, según Berlin (1977), desde el siglo XVIII.

Este autor señala que en 1725 Giambattista Vico ya establecía una diferencia entre una formación humanista, la de una mente literaria e intuitiva, poseedora de perspicacia imaginativa, y una formación en ciencias naturales que requerirá de una mente analítica y científica. Diferencia que puede equipararse con el debate recurrente que Gregory identifica entre ciencia y cultura (1962); con lo que Savater (1999) describe como lo racional y lo razonable respectivamente; o bien con la dicotomía entre humanidades-ciencias sociales y ciencias naturales, señalada por Wallerstein como "las principales divisiones del conocimiento contemporáneo" (1996, p. 54).

En este sentido, y retomando la diferenciación de que habla Gregory al hablar de "ciencia y el resto" (1962, p. 46), se confirma que no se trata solamente de una "guerra entre las ciencias" con problemáticas de diferencias en campos y metodologías; o de posiciones en contra o a favor del constructivismo social; sino de un rompimiento que viene de más atrás, de más profundo: de la identificación de ese "resto" con la cultura tradicional, y en general con la literaria, y de su comprensión como en posición antagónica con la realidad estudiada por las ciencias naturales y aquellas denominadas exactas.

Hoy en día son varias las propuestas que intentan solucionar la separación entre estas dos culturas a través de la difusión y de un proceso de comprensión pública de la ciencia; conforme a una apuesta en medios académicos especializados por articular a la ciencia y la cultura como un todo, en un momento histórico donde la actividad científica es ya parte de las prácticas diarias; donde la ciencia es la forma más poderosa de conocimiento en términos de su efecto en la vida diaria y las estructuras sociales. 
Augurada por el mismo C.P. Snow en un ensayo posterior - Las dos culturas y un segundo enfoque (1963)- y definida por John Brockman (Alvarez, 2006) como la tercera cultura, emerge un diálogo que pretende entretejer las artes y las ciencias, las ciencias sociales y las humanidades a partir de la divulgación de la ciencia y la tecnología.

Analizar las prácticas de la ciencia y de las y los científicos: factores de diferenciación con carácter eminentemente epistemológico como la naturaleza de los objetos de estudio, los campos y la estructura del conocimiento, las metodologías; y otros aspectos relacionados con cuestiones económicas y de poder porque, sin duda, la actividad científica no puede considerarse separada de las estructuras políticas y económicas que la enmarcan, resultan sumamente útiles cuando se profundiza en la comprensión pública de la ciencia.

Y para ello, la acción donde hay que ubicarse es la comprensión, porque ella no se limita a la información sino que tiene que ver directamente con el entendimiento entre las disciplinas.

No se pretende, por tanto, asumir una postura reduccionista que borre toda la especificidad de los fenómenos que pertenecen al dominio de cada disciplina, ni la noción de rigor de sus especialidades; sino de iniciar un análisis que asiente la posibilidad de reducir esas barreras de comunicación a través del conocimiento y comprensión de las lógicas de otras disciplinas sin que ello obligue a compartirlas.

\section{El carácter sociocultural del discurso disciplinario}

Partiendo de que el conocimiento está ligado a la concepción del mundo en general, y al conocimiento histórico de la disciplina en particular, es claro que el recuento histórico del surgimiento y establecimiento de las ciencias pone de manifiesto que las diferencias obvias entre las disciplinas que conforman las grandes divisiones del conocimiento de las que se viene hablando, y las barreras conceptuales y metodológicas que suelen establecerse entre ellas, no son reducibles a una oposición intrínseca a su naturaleza y deben buscarse en las formas en que fueron estableciéndose como tales y en la función que dicha fragmentación ha cumplido en diversos contextos.

El enfoque tradicional de la historia de la ciencia consideraba a las disciplinas como arquetipos predeterminados que el desarrollo del conocimiento permitía ir diferenciando de la confusión predominante en una fase pre-científica. En oposición a dicha concepción, actualmente se reconoce que el carácter de esas disciplinas científicas está determinado por 
y es contingente a la historia; toma lugar en contextos sociales e intelectuales particulares; tienen fronteras que no están predeterminadas sino que dependen tanto de las condiciones de su constitución como del desarrollo de sus relaciones con otras disciplinas igualmente contingentes histórica y geográficamente.

Esas historias juegan un papel importante en la constante reestructuración de las áreas del conocimiento, ofreciendo a las científicas y los científicos una imagen propia, de la comunidad a la que pertenecen y del propósito de su trabajo. Su historia también proporcionó a las disciplinas formas de hacer y difundir los mitos e ideologías que dieron cohesión a sus comunidades científicas: quiénes fueron sus fundadores y sus figuras destacadas, la dignidad del ramo, las metas y la relevancia social de su labor, las relaciones de cooperación y conflicto con otras disciplinas y subdisciplinas.

De esta manera y desde la perspectiva sociocultural, en particular desde la teoría de la acción mediada, no hay forma de no estar culturalmente situados cuando llevamos a cabo una acción al interior de estas colectividades, y deberíamos considerar "a los agentes, sus acciones dirigidas a metas con ciertos propósitos definidos, el escenario en donde se desarrollan tales acciones y los fines que los motivan, así como los instrumentos que utilizan para la comunicación" (Martínez, 2006, pp. 15-23).

Podría también hablarse del proceso de apropiación, que el mismo Martínez define como "activo, social y comunicativo" (2006, p. 9); y del proceso mismo de pensamiento que no debe ser

apartado de la amplitud de la vida, de las necesidades e intereses personales, de las inclinaciones e impulsos del que piensa ... [y] demuestra la existencia de un sistema dinámico de significado en el que se unen lo afectivo y lo intelectual. (Vigotsky, 1934, p.55)

Más allá de atributos y prácticas culturales que puedan distinguirlas, las características cognitivas y sociales de las disciplinas revelan una dimensión profunda de cultura, de manera que cuando hablan sobre los fenómenos que estudian y los métodos que utilizan, inevitablemente lo hacen en términos de su propia y particular representación de los mismos - lo social y cultural no solo afecta el contenido del pensamiento, sino también su método (Vigotsky, 1934).

"Todos los datos son seleccionados de la realidad con base en las visiones del mundo o los modelos teóricos de la época, filtrados por medio de las posiciones de grupos Volumen 9, Número Especial, Año 2009, ISSN 1409-4703 
[comunidades disciplinares] particulares" (Wallerstein, 1996, p. 99). La percepción nunca opera en aislamiento y sin contexto, sino en relación con el mundo social en que se desenvuelve, y dirigida por los intereses de la comunidad de la que forma parte.

Así, la objetivación de los fenómenos no es inherente a éstos ni exclusiva de nuestro aparato perceptivo, sino intrínseca a la vida social en la que juega un papel fundamental "la mediación semiótica, es decir, la interiorización de signos e instrumentos que han sido definidos culturalmente" (Martínez, 2006, p. 23).

La significación que permite una disciplina da lugar a la aparición de varias versiones del conocimiento, donde las organizaciones sistemáticas de los objetos de conocimiento son también construcciones, no reflejos de una estructura conceptual previa e independiente, ni imágenes de una realidad organizada por sí misma, sino orientada por los valores particulares de la disciplina en cuestión y dispuesta de acuerdo con la sistematización de la teoría que los analiza.

Es desde la cultura, en este caso la disciplinar, desde donde se crea un campo referencial que presta significado a un fenómeno, un objeto o una relación en el mundo experimentado, de acuerdo con condiciones de pertinencia que permiten darle un sentido del que derivará su capacidad simbólica:

Los artefactos que componen la herencia cultural material ... no sólo sirven para facilitar los procesos mentales, sino que los moldean y los transforman. Las funciones psicológicas comienzan y permanecen cultural, histórica e institucionalmente situadas y son específicas del contexto. En este sentido, no hay forma de no estar culturalmente situados cuando llevamos a cabo una acción (Martínez, 2006, p. 23).

Y es a partir de este carácter sociocultural del pensamiento, que se pretende ubicar a las disciplinas como integradoras de marcos intersubjetivos por medio de los cuales se construyen órdenes y significados. Es decir, referirse a las disciplinas como culturas que, en el caso de la y el docente universitarios, derivan en sistemas orientadores diferenciables del tipo de prácticas de enseñanza que eligen para la programación y el desarrollo de su clase.

\section{El discurso disciplinario de la enseñanza superior}

El éxito para realizar la transición está usualmente asociado con un cierto estilo de pensamiento. El filósofo F.W. J. Von Schelling introdujo la distinción (hecha famosa por Volumen 9, Número Especial, Año 2009, ISSN 1409-4703 
Nietzche) entre "apolíneos" quienes favorecen la lógica, el enfoque analítico y una evaluación desapasionada de la evidencia, y "dionisiacos" quienes se inclinan más hacia la intuición, la síntesis y la pasión ... Pero algunos de nosotros parecemos pertenecer a otra categoría: la de los "odiseicos"3 quienes combinan las dos predilecciones en su lucha por encontrar conexiones entre las ideas. Estas personas normalmente se sienten aisladas en instituciones convencionales ... (Gell-Man, 1996).

Sin embargo, los estilos de pensamiento son mucho más que meros rasgos de personalidad. A partir de la extendida convicción del carácter social del pensamiento y la cognición, Ludwik Fleck sostiene que las colectividades detentan estilos de pensamiento comunes (invariablemente relacionados con su historia); producto de la educación, tanto teórica como práctica, que recibe el individuo a través de experiencias de diversa índole, y que no sólo le predisponen, sino literalmente le dictan qué y cómo ver: "los estilos de pensamiento crean la realidad de la misma manera que otros productos culturales" (1936, p.112).

En el caso de la "colectividad" disciplinaria, la naturaleza comunitaria de la cognición científica se hace obvia; tanto por su estructura y características como por la singularidad histórica de su desarrollo. Como acto social, se basa en conocimientos y habilidades transmitidos a los nuevos miembros como capital de dicha colectividad, y no de un autor o autora en lo individual; construye lazos entre los integrantes y "ciertamente determina todo acto de cognición" (Fleck, 1960, p. 154).

Esta conclusión no es exclusiva de la escuela vigotskyana; desde los procesos culturales, el planteamiento coincide con los esquemas de percepción y apreciación que guían prácticas concordantes y constituyen el habitus en el sentido en que Andión (1992) describe la noción de Pierre Bourdieu. Desde la sociología de la ciencia puede leerse también a la luz de la inconmensurabilidad de los paradigmas (Kuhn, 1971), ya que de acuerdo con Fleck (1935) un pensamiento cobra sentido en la medida en que encaja en el sistema propio de un estilo.

Desde lo educativo, además de cumplir la función de "disciplinar las mentes y canalizar la energía de los estudiantes" (Wallerstein, 1996, p.103), estaríamos hablando de un entrenamiento diferenciado de la percepción hacia las formas específicas que cada campo disciplinario considera esenciales; porque para Fleck (1935), hasta la más sencilla

\footnotetext{
${ }^{3}$ Traducción libre.
} 
observación (observar los experimentos demostrativos en el ámbito escolar, por ejemplo) requiere cierta disposición mental, por lo que una gran parte de la educación disciplinaria consiste precisamente en enseñar al estudiantado a ver lo que sus docentes ven.

En su forma de enseñar, el grupo docente enfatizará información concreta (hechos) o abstracta (conceptos o teorías); utilizará mayormente presentaciones visuales (diagramas, demostraciones, ilustraciones) o verbales (exposición, dictado, discusiones); organizará la presentación inductivamente (el fenómeno lleva a los principios) o deductivamente (los principios llevan al fenómeno); favorecerá en su clase la participación activa del alumnado o le asignará un papel pasivo, exclusivamente de recepción; y su perspectiva de acercamiento a la materia será secuencial o global. En síntesis, la combinación que la y el docente haga de estos elementos estará altamente determinada por la naturaleza misma de la disciplina que imparte y de cuya cultura participa.

Basado no sólo en factores cognoscitivos, sino en el cruce entre aspectos epistemológicos y sociológicos, y en una exhaustiva revisión de clasificaciones y taxonomías de aceptación generalizada principalmente en el ámbito anglosajón, el investigador inglés Tony Becher (1989, 1993) establece los siguientes cuadrantes:

\section{Disciplinas Duras/Puras}

Caracterizadas principalmente por la naturaleza acumulativa del conocimiento; donde hay una relativa progresión lineal; problemas subdivisibles y atacables individualmente; que exponen regularidades y dan importancia al descubrimiento.

\section{Disciplinas Blandas/Puras}

Caracterizada por asuntos básicamente recurrentes; problemas multifacéticos y no fácilmente divisibles; preocupadas por las instancias particulares; cuyo producto final típico son la comprensión y la interpretación.

\section{Disciplinas Blandas/Aplicadas}

Que interpretan el conocimiento blando/puro para entender y manejar las condiciones de complejidad de las situaciones humanas; cuya efectividad es juzgada por criterios funcionales y de utilidad. Como su preocupación está en realizar la práctica, sus resultados son protocolos y procedimientos. 
Por supuesto esta y otras clasificaciones deben verse como estrategias convenientes, pero contingentes, para ordenar lo que investigado acerca de cómo se manifiestan los procesos relacionados con el conocimiento y su transmisión; pero no constituyen categorías naturales por lo que los límites entre los dominios no pueden establecerse con precisión ${ }^{4}$.

Sin intención alguna de caer en estereotipos y asunciones ligeras, y exhortando a las y los lectores a no hacerlo, a continuación se describen con más detalle cada uno de los cuadrantes de esta tipología, con base en la información que al respecto arrojan las investigaciones de carácter empírico consultadas a las que se irá haciendo referencia conforme corresponda; advirtiendo que el presente análisis se concentra en el nivel de las carreras de grado porque, basada en un serio estudio del estado del arte, Neumann (2001, p. 137) declara que "resulta considerable la variación en los métodos y estilos de enseñanza entre este nivel y los característicos de los posgrados". Fue necesario dejar fuera también, otros aspectos como los referentes a cuestiones propias de la actividad científica y los dominios de investigación correspondientes, los cuales difieren de sus prácticas docentes según la misma autora; y sería recomendable mantener presente, que se hace referencia a la formación profesional y no al desarrollo de disciplinas científicas.

La investigación actual demuestra la fuerza de las creencias del profesorado sobre las materias que imparte y la dificultad existente en modificarlas (Stodolsky y Grossman, 1995). Hace evidente también, como a un énfasis en metas diferentes corresponden estilos de enseñanza que reflejan las creencias pedagógicas de las diversas disciplinas, y, por tanto, las prácticas didácticas y aquello que se exige del alumnadp, reflejarán también esas creencias y metas (Neumann , 2001). Gran parte del conocimiento pedagógico específico de las disciplinas es más bien implícito, relacionado con lo que el cuerpo académico sostiene como fundamentos de la enseñanza en sus campos, y constituye las bases para comprender las razones del proceso de enseñanza adoptado (Neumann, 2001).

Tratando de ir de lo general a lo particular, a continuación se relacionan algunas de las características a las que Becher $(1989,1993)$ hace alusión para cada uno los cuadrantes de su tipología, con los estilos de enseñanza particulares que los distinguen y que, es la intención de este análisis evidenciar, se desprenden lógicamente de ellas.

\footnotetext{
${ }^{4}$ De acuerdo con Tony Becher (1989, p. 151) "incluso la disciplina aparentemente más blanda tiene bordes duros, y los márgenes de las duras permiten una buena cantidad de blandura". Volumen 9, Número Especial, Año 2009, ISSN 1409-4703
} 
Ya que las disciplinas Duras/Puras típicas (aquellas fuertemente paradigmáticas), se caracterizan por estar bien estructuradas, porque el terreno de estudio de los fenómenos es limitado, los métodos son estrictamente definidos y la investigación es altamente reproducible; puede decirse que el profesorado aprecia altamente la coherencia en el currículum, pero no así la idea de perspectivas críticas. Mientras que docentes de las disciplinas Blandas/Puras muestran precisamente el patrón contrario, buscando desarrollar en su alumnado perspectivas críticas dada su aceptación de paradigmas múltiples, lo cual a su vez los hace renuentes hacia el tema de la coherencia curricular (Neumann , 2001); de forma congruente con su carácter de no restringidas, porque su campo o fenómenos son relativamente ilimitados, “con métodos y contenidos más idiosincráticos” (Donald, 1995, p. 7) y porque consideran la complejidad como un aspecto legítimo del conocimiento.

Las diferentes disciplinas parecen enfocarse en aspectos particulares del proceso de pensamiento crítico; un ejemplo para las Duras/Puras lo constituye el proceso inferencial en la física. Las ciencias sociales, como Blandas/Puras que son, tienden a interpretar las características del método científico -replicabilidad, demostración empírica, naturaleza autocorrectiva- de manera diferente al de las primeras (Donald. 1995).

En el cuadrante Duro/Puro se combina una gran confianza en la experiencia con la teorización sistemática, mientras en las humanidades, del lado Blando del espectro, se confía en la experiencia pero no en un código específico de razonamiento. Las matemáticas y la lógica serían independientes de la experiencia, pero altamente estructuradas y sistemáticas y por ello corresponden al primer tipo.

Aunque la consistencia aparece como un criterio importante en todas las disciplinas, en las Blandas/Aplicadas hablaríamos de una consistencia en el tiempo y entre personas, mientras que en las Blandas/Puras la consistencia toma la forma de confiabilidad sobre una serie de observaciones. La precisión y la exactitud pueden ser consideradas como una medida directa de estructura y codificación en las áreas Duras, pero en dominios menos estructurados o más complejos, la coherencia del argumento o la completud o comprensibilidad de la teoría resultan más importantes.

Desde esta perspectiva y considerando que aquello que se exige del alumnado refleja también los objetivos y las metas favorecidos particularmente por las disciplinas, se sigue que el grupo docente de las Duras tendería a probar la experiencia contra la teorización sistemática, y el de las Blandas/Puras intentaría seguir el mismo patrón en determinado nivel, pero sin desplegar el mismo grado de estructura o especificidad que el esperado en las 
primeras. El profesorado de las Blandas/Aplicadas no estaría en posición de probar la experiencia contra teorías sistemáticas, pero estaría interesado en la prueba del tiempo, como medio de certificación de la experiencia (Donald, 1995).

En otro aspecto de la legitimación, las investigaciones del mismo Donald (1995) sostienen que los métodos en las humanidades, del lado Blando de la tipología adoptada, son la hermenéutica y el pensamiento crítico, mientras que en las disciplinas Duras los métodos serían la solución de problemas y el método científico. No obstante, en lo correspondiente a los procesos de validación, parece haber una interacción entre Dura y Blanda / Pura y Aplicada, donde miembros de las disciplinas Puras le dan mayor reconocimiento e importancia al proceso.

Antes de abandonar lo correspondiente a creencias y fundamentos para pasar a cuestiones más concretas, conviene agregar que si bien se señalaba que dichas creencias son difíciles de modificar, John M. Braxton concluye de sus estudios que las y los académicos de las disciplinas Blandas son más afines a las reformas educativas, y que son "aliados(as) naturales de cambios y mejoras en los currículums de carreras de grado" (1995, p. 60), lo cual debe tener que ver con algunas de las características ya mencionadas de las que habla Becher, relativas a límites internos débiles que además de permitir el movimiento a través de ellas con cierta facilidad, las hace menos resistentes al cambio (1989).

Para continuar, resulta importante enfatizar la distinción entre las dimensiones Puras y las Aplicadas. Las disciplinas Puras son aquellas que desarrollan su propio modelo o paradigma (por ejemplo la física y las matemáticas del lado Duro del cuadrante, o la sociología del Blando), mientras que los "campos aplicados" ${ }^{5}$ son aquellos que usan, y expanden en la práctica, el modelo o paradigma trabajado por las Puras (por ejemplo la Educación o el Derecho). Esta distinción establece de entrada una diferencia significativa entre los objetivos educativos a perseguir; diferencia que se extenderá al cruzar esta dimensión de lo Puro/Aplicado con la de lo Duro/Blando.

Si presuntamente las disciplinas Duras/Puras buscan construir teorías poderosas y generales, en su investigación empírica Cashin y Downey (1995) reportan que el 100\% del profesorado de estas áreas consideró más importante que el resto del profesorado de su muestra, el que la población de estudiantes adquiriera conocimiento factual, definido como

\footnotetext{
${ }^{5}$ Llamados así precisamente porque "los fenómenos que estudian son relativamente irrestrictos y sus
} métodos son diversos" (Donald, 1995, p. 8) 
terminología, clasificaciones, métodos y tendencia; y que el grupo estudiantil afirma estar aprendiendo especialmente aquello que sus docentes consideran más importante y que, podemos inferir, enfatizan en su enseñanza.

En cambio, si para las Duras/Aplicadas la función primordial del conocimiento es la generación de técnicas orientadas a los productos y de productos en sí, la preocupación principal de profesores y profesoras del área será la preparación profesional de su alumnado, como lo demuestran empíricamente Gaff y Wilson (Braxton, 1995).

Otra diferencia al interior de las disciplinas Duras que dependerá de esta dimensión Pura/Aplicada, es la interpretación del concepto de "aplicación" de una teoría. Por ejemplo, una disciplina Dura/Pura, como la física, se referiría para el caso a la utilización de las derivaciones matemáticas en la resolución de problemas; mientras que una Aplicada, como la ingeniería, entendería por ello la comprensión de la manera en que funcionan los dispositivos electrónicos (Hativa, 1995, y Neumann, 2001).

Si en ambos casos, como sostiene Braxton (1995), se tiende a examinar al estudiantado en cuestiones que requieren un mayor grado de memorización y aplicación de los contenidos; del lado de las disciplinas Blandas este autor distingue en cambio preguntas o tareas que requieren análisis o síntesis de los contenidos de los cursos, lo cual coincide ampliamente con un tipo de conocimiento altamente heterogéneo, que comprende el estudio de lo particular más que de lo general y busca la comprensión y la interpretación más que la explicación causal, aunque aspire a cierto grado de generalidad en la investigación de sus ramas Puras.

El énfasis en la participación del alumnado en discusiones y diálogos y en abarcar diversas perspectivas, al que el mismo Braxton se refiere como un "enfoque discursivo" (1995, p. 60), donde el profesorado de las áreas Blandas abre la discusión a puntos de vista que no son los propios; atiende a cuestiones más allá de las establecidas en el temario; y relaciona los asuntos del curso con otros campos de estudio, puede considerarse un reflejo de los elementos constitutivos del conocimiento Blando, cuyo desarrollo es recursivo o reiterativo (en contraposición al acumulativo y relativamente sostenido de las disciplinas Duras), y donde son los problemas los que determinan los métodos (Becher, 1989).

Retomando esta última característica del conocimiento en las áreas Duras, su carácter acumulativo y fragmentable, puede comprenderse el concepto de "conocimiento más substancial' al que Hativa (1995, p. 26) se refiere cuando habla de la mayor cantidad de conocimientos previos requeridos por las disciplinas Duras/Puras. 
Tanto el grado de seriación como la cantidad de materias que constituyen un prerrequisito para estudiar otras posteriormente, parecen tener que ver también con la naturaleza de la disciplina. Stodolosky y Grossman acumularon evidencias de que los profesores de matemáticas, en comparación con los de otros campos, tienen menos control sobre lo que enseñan y se atienen a un currículum más estandarizado en términos de contenidos y hasta de exámenes comunes, y citan a uno de ellos haciendo alusión a este tipo de prerrequisitos: "No puedes cambiar los temas, porque mucho en álgebra depende de lo que hayas aprendido con anterioridad. En buena medida, no puedes pasar a resolver problemas si no has trabajado antes con números positivos y negativos" (1995, p. 74).

Esto explica por qué si diversos estudios demuestran que el conocimiento Duro se desactualiza más rápidamente que el Blando (Becher, 1989), en la configuración cognoscitiva de la enseñanza de las disciplinas, en el extremo Blando del espectro las profesoras y los profesores proporcionan al alumnado conocimientos más recientes (especialmente cierto para el caso de las ciencias sociales). Para Neumann (2001), esta práctica parece estar relacionada con la estructura jerárquica del conocimiento en las disciplinas Duras, donde lo más actualizado sólo se introduce en los posgrados.

Como afirman Rodriguez Sala y Tovar, "con frecuencia los fenómenos de las ciencias exactas [disciplinas Duras], en su descripción y análisis, resultan difíciles de expresar con las formas lingüísticas usuales y muy a menudo se requiere la creación de nuevos términos o bien una composición gramatical propia" (1982, p. 91), a los que alumnos y alumnas tendrán que ser paulatinamente introducidos. Esto es congruente con la afirmación de Vigotsky con respecto a los conceptos científicos que: "no se asimilan tal cual" (1934, p. 163), y en cuya adquisición la enseñanza y el aprendizaje juegan un papel importante.

Y habría que recordar también la perspectiva de T.S. Kuhn (2001), quien considera característico de ciencias maduras (Duras en este caso) el que los conocimientos se basen en libros de texto, los cuales constituyen la fuente de autoridad y el vehículo pedagógico para enseñar a la población estudiantil lo que su comunidad científica contemporánea acepta como verdadero, y para comunicarle el vocabulario y la sintaxis de su lenguaje especializado.

De esta manera, y reflejando de nuevo los elementos constitutivos del tipo de conocimiento de que se trata, al abordar la enseñanza de sus disciplinas, las áreas Duras requerirán mayor memorización y aplicación del material del curso, mientras las disciplinas Blandas tenderán a examinar mediante preguntas que impliquen análisis y síntesis de los 
contenidos. En cuestiones más concretas que pueden servir de ejemplo, y de acuerdo con las y los autores revisados, aunque todas las disciplinas a nivel de licenciatura (carrera de grado) hagan uso frecuente de exámenes escritos, los reportes basados en trabajo práctico son usados sólo en los campos Puros, mientras que las presentaciones orales son características de los Blandos/Aplicados. En un estudio detallado y a gran escala, Warren Piper et al. (Neumann, 2001) lograron establecer que las disciplinas Duras, Puras y Aplicadas, otorgan un peso mayor a los exámenes estructurados que las Blandas, las cuales utilizan en mayor medida ensayos, exámenes con preguntas abiertas y reportes de proyectos.

Lo anterior está directamente relacionado con el papel que los estilos de pensamiento de las diversas comunidades confieren al alumnado al abordar su educación. En lo correspondiente a las prácticas didácticas, las profesoras y los profesores del extremo Blando tienden a mostrar un rango más amplio de actividades.

Los resultados empíricos hallados por Murray y Renaud (1995) apuntan a una promoción más generalizada del papel activo y participativo de alumnos y alumnas en clase por parte de las artes y humanidades (ambas en el cuadrante Blando/Aplicado), mientras el grupo docente de ciencias naturales y demás disciplinas Duras, muestran más frecuentemente comportamientos que favorecen la estructura y organización de la materia de estudio. Siguiendo lo reportado por estos autores, en una investigación donde se comparan profesores en términos de objetivos instruccionales y prácticas de evaluación, parece evidenciarse que las profesoras y los profesores de las disciplinas Blandas/Aplicadas tienden a enfatizar metas relacionadas con el "pensamiento" más que las vinculadas con los "hechos", y a favorecer la discusión y los proyectos independientes más que la exposición por parte del profesorado. Así mismo, Stodolsky y Grossman (1995) reportan que en las disciplinas Duras/Puras la población docente se interesa mayormente por niveles de logro absolutos; mientras que quienes enseñan en las Blandas atienden también al esfuerzo y la participación.

Que el pensamiento deductivo en la enseñanza de las áreas Duras parta de un modelo claramente postulado, corresponde al ideal metódico relativamente fácil de delinear de estas disciplinas y, por tanto, es de esperarse la falta de referencia al mismo en las áreas Blandas, donde no es fácil identificar modelos de esta naturaleza.

En este sentido, y sin referirse explícitamente a lo inductivo/deductivo, Kuhn (2001) hace un señalamiento similar cuando habla del conocimiento tácito, advirtiendo sobre la 
problemática contradicción existente entre la creencia de profesores y profesoras de que el alumnado en clase o laboratorio no puede resolver problemas o realizar ejercicios a menos de que conozca con anterioridad la teoría y algunas reglas para su aplicación, mientras el mismo Kuhn (2001) establece que el aprendizaje realmente se adquiere practicando la ciencia y no mediante reglas para practicarla.

Para concluir el apartado y a manera de síntesis, el siguiente cuadro recoge las diferencias antes detalladas. Los cinco aspectos son sólo una forma de organizar los hallazgos de las investigaciones citadas y se encuentran significativamente interconectados entre sí:

\begin{tabular}{|c|c|c|}
\hline ASPECTO & DISCIPLINAS DURAS & DISCIPLINAS BLANDAS \\
\hline Conocimiento & $\begin{array}{l}\text { Factual, terminología, } \\
\text { clasificaciones. } \\
\text { Acumulativo y fragmentable. } \\
\text { Lenguaje especializado. } \\
\text { Busca explicación causal. Metas } \\
\text { relacionadas con los hechos. } \\
\begin{array}{l}\text { Se desactualiza rápidamente. } \\
\text { Estructura jerárquica de los } \\
\text { conocimientos. }\end{array}\end{array}$ & $\begin{array}{l}\text { Más de lo particular. } \\
\text { Heterogéneo. } \\
\text { Gama más amplia de lenguajes. } \\
\text { Busca comprensión e } \\
\text { interpretación. Metas relacionadas } \\
\text { con el pensamiento. }\end{array}$ \\
\hline $\begin{array}{l}\text { Estructura } \\
\text { Currículo }\end{array}$ & $\begin{array}{l}\text { Estandarizado. Valora coherencia. } \\
\text { Mayor resistencia a los cambios } \\
\text { por lo estructurado de sus límites } \\
\text { internos. } \\
\text { Mayor cantidad de conocimientos } \\
\text { previos requeridos. } \\
\text { Mayor grado de seriación. }\end{array}$ & $\begin{array}{l}\text { Poca coherencia curricular. Valora } \\
\text { perspectivas críticas. } \\
\text { Afines a las reformas educativas. } \\
\text { Mayor apertura a la discusión de } \\
\text { temas fuera de los contenidos } \\
\text { establecidos. }\end{array}$ \\
\hline \multirow[t]{2}{*}{ Método } & $\begin{array}{l}\text { Estrictamente definido. } \\
\text { Inferencia, replicabilidad. } \\
\text { Demostración empírica. } \\
\text { Consistencia entre serie de } \\
\text { observaciones. } \\
\text { Teorización sistemática. } \\
\text { Precisión y exactitud. } \\
\text { Validación* a través de solución } \\
\text { de problemas y del método } \\
\text { científico. }\end{array}$ & $\begin{array}{l}\text { Enfoques y perspectiva varios. } \\
\text { Los problemas determinan los } \\
\text { métodos. Interpretación. } \\
\text { Consistencia en tiempo y entre } \\
\text { personas. } \\
\text { Completud o comprensibilidad. } \\
\text { Validación * a través de la } \\
\text { hermenéutica y el pensamiento } \\
\text { crítico. }\end{array}$ \\
\hline & \multicolumn{2}{|c|}{$\begin{array}{c}\text { *En ambos casos, los procesos de validación son más importantes } \\
\text { para las disciplinas Puras que para las Aplicadas. }\end{array}$} \\
\hline
\end{tabular}




\begin{tabular}{|c|c|c|}
\hline $\begin{array}{l}\text { Papel de } \\
\text { alumnos }\end{array}$ & $\begin{array}{l}\text { Mayor memorización } \\
\text { aplicación* de los contenidos. } \\
\text { Exposiciones estructuradas. }\end{array}$ & $\begin{array}{l}\text { Procesos de análisis y síntesis de } \\
\text { los contenidos. } \\
\text { Participación activa del alumno. } \\
\text { Rango más amplio de actividades. }\end{array}$ \\
\hline & \multicolumn{2}{|c|}{$\begin{array}{l}\text { *Utilización de teorías en la resolución de problemas para las Puras, } \\
\text { Comprensión del funcionamiento de dispositivos para las Aplicadas. }\end{array}$} \\
\hline $\begin{array}{l}\text { Formas } \\
\text { Evaluación }\end{array}$ & $\begin{array}{l}\text { Metas relacionadas con los } \\
\text { hechos. } \\
\text { Niveles de logro absoluto. }\end{array}$ & $\begin{array}{l}\text { Presentaciones } \\
\text { (principalmente en el caso de las } \\
\text { Aplicadas) } \\
\text { Metas relacionadas con el } \\
\text { pensamiento. } \\
\text { Valora esfuerzo y participación. }\end{array}$ \\
\hline
\end{tabular}

\section{Conclusiones: en busca de nuevas articulaciones}

"The habit of active thought with freshness can only be generated by adequate freedom. Undiscriminating discipline defeats its own object by dulling the mind"6

A.N. Whitehead

Probablemente esta cita se refiere a una de las acepciones de disciplina que aparecen en el diccionario, relativa a la sujeción a normas severas. Aquí se incluye por considerarse que bien puede aplicarse en este caso al empleo que, de manera bastante poco estricta, se ha dado en este ensayo al concepto de campos (en los sentidos sociológico y epistemológico) circunscritos en la actividad académica.

$Y$ es que sin pretender asumir una postura reduccionista que borre toda la especificidad de los dominios de cada disciplina, ni la noción de rigor de sus especialidades, se considera que no puede pasarse ya por alto que se trata de la formación de sujetos en y para un mundo donde se están cayendo a pedazos los paradigmas de la modernidad (racionalidad instrumental, enfoque positivista de la ciencia, etc.), y del otro lado parece no haber nuevos asideros; donde ha tenido lugar una ruptura irreversible de los horizontes que se tenían por absolutos. Y donde una de las pocas certezas que prevalecen es que el diálogo interdisciplinar está en la esencia del progreso intelectual.

${ }^{6}$ El hábito del pensamiento activo e inédito solo puede ser generado por una adecuada libertad. La disciplina indiscriminada traiciona su propio objetivo entorpeciendo la mente (traducción libre). En: http://www.uky.edu/ eushe2/quotations/whitehead.html 
Por ello, estas conclusiones se abocan en primer lugar a la necesidad de estudiar más los diversos grupos disciplinarios en relación con los mundos educacionales que habitan. Solo si se comprende la naturaleza específica de sus contenidos y sus metas particulares podrá mejorarse significativamente la enseñanza y orientar el diseño curricular; porque, valga insistir, esos mundos suponen un concepto de enseñanza/aprendizaje implícito, así como un conjunto de procesos interpretativos que atraviesan la construcción de un uso diferenciado de métodos de enseñanza por parte de las disciplinas, que confiere a los mismos diversos significados a partir de los marcos de referencia y los entornos de interpretación en que se realiza la interacción con ellos.

En segundo lugar, caber reiterar el énfasis que la y el docente hace, tanto explícita como implícitamente, en aquello que su disciplina considera importante, no sólo en términos de contenidos, sino de la naturaleza de las metas que se plantea y de aquello que exige de alumnos y alumnas.

Las intenciones y resoluciones de profesoras y profesores coinciden con los campos específicos del conocimiento que cultiva, y con las tradiciones de la comunidad disciplinaria a la que pertenecen. Es precisamente de las características epistemológicas y sociológicas de esas culturas disciplinarias, constituidas en verdaderos sistemas, de las que se desprenden sus prácticas pedagógicas concretas.

La reflexión realizada en este ensayo ejemplifica dicha concordancia, y pone también en evidencia algunos cuestionamientos importantes para la educación superior:

- Cómo incorporar en sus programas una ampliamente compartida recomendación hacia la flexibilidad ${ }^{7}$ en la formación de futuros profesionistas que deberán adaptarse a las demandas de un entorno constantemente cambiante.

- Cómo balancear las presiones contrarias de la especialización, por una parte, y de perspectivas en pro de la integración de los saberes, por otra.

- Y en este mismo sentido, cómo atender desde la educación superior el cuestionamiento sobre la estructura disciplinaria; no tanto en lo administrativo, sino en lo relativo a la ampliación de la organización de la actividad intelectual (Wallerstein, 1996).

${ }^{7}$ La Conferencia Mundial de la UNESCO (1998) sobre la Educación Superior en el Siglo XXI, habla de: amplia movilidad ocupacional, multifuncionalidad, polivalencia, interdisciplinariedad, trabajo en equipo, movilidad geográfica y nuevas formas de relación laboral, como características de la Volumen 9, Número Especial, Año 2009, ISSN 1409-4703 
- Cómo contrarrestar los perniciosos efectos de esquemas que devienen poco tolerantes: "Cada colectividad de pensamiento considera incompetente a quien no pertenece a ella" (Fleck, 1946, p. 127); y que en muchos casos se traducen en prejuicios y prácticas absurdas que ponen en riesgo el éxito estudiantil de algunas personas (con respecto a la identificación de ciertos estilos y habilidades con una disciplina en particular, y la forma en que con estos preconstruidos la y el docente "mide" a estudiantes que pudiesen ser diferentes).

- De qué manera podemos, como sugiere Fleck (1935), dar un giro en la educación que nos permita ver los condicionamientos [disciplinarios], y cómo han sido determinados históricamente, con objeto de crear otras predisposiciones mentales [de mayor apertura] y educar a las personas a vivir en ellas.

Si no podemos saltar por encima de nosotros mismos; si siempre cargamos con nuestra situación histórica, nuestra experiencia concreta, y el horizonte comprensivo desde el cual damos sentido a los fenómenos particulares (Coreth, 1985); consideremos, de entre nuestras pertenencias múltiples, la disciplinaria en una sincera preocupación por abrir nuestra preconcepción en tanto que tal.

La perspectiva histórico culturalista encabezada por Vigotsky, al hacer evidente que pensamiento y lenguaje tienen un carácter eminentemente social: social en sus orígenes, social en sus funciones; social en su forma y social en sus aplicaciones; abre posibilidades esperanzadoras al diálogo interdisciplinar y, en general, a un reconocimiento de la pluralidad que favorece la comunicación y la integración de saberes porque pone en evidencia que el desarrollo psicológico no es exclusivamente cognitivo y por ello inalterable, sino una expresión del desarrollo cultural.

Ante el cuestionamiento de que son objeto las fronteras disciplinarias, se impone la redefinición de mapas culturales y cognoscitivos: hay que conocer/aprender más, pero también y sobre todo, hay que conocer/aprender de forma diferente.

Y es en esta búsqueda de nuevas formas de articulación, una de las preocupaciones que flotan en el ambiente en varias dimensiones (lingüísticas, históricas, sociales, etc.), que emerge el pensamiento interdisciplinario como pertinente en el caso de objetos de estudio y

ocupación profesional que la universidad del siglo XXI debe considerar en la formación de sus alumnos. 
comunidades (Fuentes, 1998) como los son las disciplinas y las profesiones. Adquiere entonces un carácter de inaplazable, la reconfiguración del sentido de la prácticas docentes ante un entorno tan rápidamente cambiante que deviene amenazante para la educación superior ante nuevos mapas laborales y nuevos modos de trabajar que exigen una actualización de lógicas y modelos de comunicación interdisciplinaria. Y, ¿por qué no? la promoción de ese estilo de pensamiento "odiséico" (citado en el apartado no. 4) como posibilidad de comunicación articuladora.

En el marco de las culturas disciplinarias, no resulta aventurado afirmar que el manejo de la diversidad está en el corazón de la educación superior. La universidad tiene que ver con lo interdisciplinario; supone una comunicación abundante (de ahí su gestión colegiada de lo académico), porque es al interior de la universidad que se da el diálogo entre los diferentes saberes sobre la realidad: es ella quien que realiza ese diálogo, pero también la que lo provoca.

No se trata sin embargo de cualquier tipo de diálogo. No siendo el cometido de este ensayo el debate de fondo sobre las diferencias conceptuales entre interdisciplinariedad, transdisciplinariedad, multidisciplinariedad, posdisciplinariedad, etc., se limita a plantear la posibilidad de una comunicación articuladora de las culturas disciplinarias no desde una complacencia relativista (que de entrada desalienta la búsqueda de puntos de coincidencia en el terreno cognoscitivo porque no acepta la viabilidad de acuerdos fuera del sistema de creencias, valores y normas de una comunidad particular); ni desde un ideal absolutista basado en un principio universalista en búsqueda de una cultura monolítica y coherente (y que supone la asimilación de todas las culturas a un único patrón "verdadero"); sino desde una perspectiva pluralista para la cual "no hay una única, correcta y completa manera de caracterizar a la ciencia, lo mismo que no puede haber una única, correcta y completa manera de representarse al mundo" y donde la idea de hechos "no es la idea de hechos absolutos cuya existencia es independiente de los recursos conceptuales, metodológicos, tecnológicos y prácticos que tengan los agentes que intentan conocer el mundo" (Olivé, 2000, p. 189).

Producto de un proceso iniciado hace varias décadas, el contexto socio-histórico impone sobre los campos disciplinarios demandas particulares y cuestiona sus objetos, metodologías y organización especialmente en lo que concierne a rígidas fronteras y a maneras poco tolerantes de encarar la diversidad en una producción y transmisión del 
conocimiento relacionada cada vez en mayor grado con esquemas de conexión o desconexión a redes.

Sin embargo, todo ello no es fácil en culturas tan fuertemente orientadas disciplinariamente. Una conceptualización más allá de las disciplinas no sólo tiene implicaciones para la formación y la discusión reflexiva sobre las profesiones; implicaciones metodológicas para el trabajo concreto; para la formulación misma de problemas. Requiere además una reconceptualización al interior de cada disciplina y plantea una problemática fundamentalmente epistemológica.

La recompensa a un esfuerzo de tal magnitud no sería despreciable. El proceso de mezcla es también fuente de enorme creatividad cultural y dinamismo. La hibridación de la cultura es la base de un trabajo más original y emocionante. Los replanteamientos y la movilidad son fuentes potentes de innovación y desarrollo, y traen consigo nuevas maneras de mirar las cuestiones conocidas (Becher, 1989) y también de descubrir oportunidades hasta ahora inexploradas.

\section{Referencias}

Álvarez Muñoz, Evaristo. (2006). La guerra de las ciencias y la tercera cultura. Chile: Red Cinta de Moebio. Recuperado en Mayo 2009 de http://site.ebrary.com/lib/iberoleonsp/Doc?id=10104711\&ppg=5.

Andión, Eduardo. (1992). Pierre Bourdieu y la comunicación social. México: UAM.

Becher, Tony. (1993). Las disciplinas y la identidad de los académicos. En Pensamiento Universitario, 1 y recuperado en Junio 2004 de http://www.argiropolis.com.ar/documentos/Becher.htm.

Becher, Tony. (1989). Tribus y territorios académicos. La indagación intelectual y las culturas de las disciplinas. España: Gedisa, Biblioteca de educación.

Berlin, Isiah. (1977). The proper study of mankind, an anthology of essays. New York: Farrar, Stauss and Giroux.

Biglan, Anthony. (1973). The characteristics of subject matter in different academic areas. Journal of Applied Psychology, 57 (3), 195-203.

Braxton, John M. (1995). Disciplines with an affinity for the improvement of undergraduate education. En Hativa, Nira y Michele Marincovich (editors), Disciplinary differences in teaching and learning: implications for practice (pp. 59-64). San Francisco: Jossey- Bass. 
Cashin, William E. y Ronald G. Downey. (1995). Disciplinary differences in what is taught and in students perceptions of what they learn and of how they are taught. En Hativa, Nira y Michele Marincovich (editors), Disciplinary differences in teaching and learning: implications for practice (pp. 81-92). San Francisco: Jossey-Bass.

Coreth, Emerich. (1985). ¿Qué es el hombre? Esquema de una antropología filosófica. Barcelona: Herder.

Davídov, Vladimir V. (1988). La enseñanza escolar y el desarrollo psíquico. Investigación psicológica teórica y experimental. Moscú: Progreso.

Donald, Janet G. (1995). Disciplinary differences in knowledge validation. En Hativa, Nira y Michele Marincovich (editors), Disciplinary differences in teaching and learning: implications for practice (pp. 7-18). San Francisco: Jossey-Bass.

Fleck, Ludwik. (1960). Crises in science. Recuperado en Junio 2004 de http://www.fmag.unict.it/polhome.html.

Fleck, Ludwik. (1946). Problems of the science of science. Recuperado en Junio 2004 de http://www.fmag.unict.it/polhome.html.

Fleck, Ludwik. (1936). The problem of epistemology. Recuperado en Mayo 2009 de http://cavanegas.googlepages.com/ParcialFilosofia.pdf

Fleck, Ludwik. (1935). Scientific observation an perception in general. Recuperado en Junio 2004 de http://www.fmag.unict.it/polhome.html.

Fuentes Navarro, Raúl. (1998). La emergencia de un campo académico: continuidad utópica y estructuración científica de la investigación de la comunicación en México. México: ITESO-Universidad de Guadalajara.

Galperin, Piotr I. (1987). Sobre la investigación del desarrollo intelectual. En M. Shuare y V. Davídov (compiladores), La psicología evolutiva y pedagógica en la URSS. Antología (pp.125-142). Moscú: Progreso.

Gell-Man, Murray. (1994). The quark and the jaguar. Adventures in the simple and the complex. U.S.A.: Plenum.

Gregory, Jane and Steve Miller. (1962). Science in Public. Communication, culture, and credibility. U.S.A.: Plenum.

Hativa, Nira. (1995). What is taught in an undergraduate lecture? Differences between a marched pair of pure and applied disciplines. En Hativa, Nira y Michele Marincovich (editors), Disciplinary differences in teaching and learning: implications for practice (pp.19-30). San Francisco: Jossey-Bass.

lanni, Octavio. (2000). Enigmas de la modernidad - mundo. México: Siglo XXI. 
Kuhn, Thomas Samuel (1971). La estructura de las revoluciones científicas. México: Fondo de Cultura Económica.

Martínez Rodríguez, Miguel Ángel. (2006). El enfoque sociocultural en el estudio del desarrollo y la educación. México: Red Revista Electrónica de Investigación Educativa, Recuperado en $\quad$ Mayo 2009 de. http://site.ebrary.com/lib/iberoleonsp/Doc?id=10122091\&ppg=10.

Murray, Harry G. y Robert D. Renaud. (1995). Disciplinary differences en classroom teaching behaviors. En Hativa, Nira y Michele Marincovich (editors), Disciplinary differences in teaching and learning: implications for practice (pp. 31-40). San Francisco: Jossey-Bass.

Neumann, Ruth (2001). Disciplinary Differences and University Teaching. Studies in Higher Education, 26 (2), 135-146.

Olivé, León (2000). Heurística, multiculturalismo y consenso. En Velazco Gomez, Ambrosio (coord.), El concepto de la heurística en las ciencias sociales y las Humanidades (pp.176-200). México: Siglo XXI/ UNAM Centro de Investigaciones Interdisciplinarias.

Rodríguez Sala, Ma. Luisa y Tovar, Aurora. (1982). El científico como productor y comunicador. El caso de México. México: UNAM.

Savater, Fernando. (1999). Valores morales y valores científicos. Ponencia marco en el I Congreso sobre Comunicación Social de la Ciencia. España.

Snow, Charles Percy. (1963). Las dos culturas y un segundo enfoque. Madrid: Alianza Editorial, Reedición 1977.

Stodolsky, Susan S. y Grossman, Pamela. (1995). Subject-matter differences in secondary schools: connections to higher education. En Hativa, Nira y Michele Marincovich (editors), Disciplinary differences in teaching and learning: implications for practice, (pp.71-80). San Francisco: Jossey-Bass.

Vigotsky, Lev. (1934). Pensamiento y lenguaje. España: Paidós.

Wallerstein, Immanuel (coord.). (1996). Abrir las ciencias sociales. México: Siglo XXI-CIIH, UNAM.

Wallerstein, Immanuel. (1998). Conocer el mundo, saber el mundo: el fin de lo aprendido. Una ciencia social para el siglo XXI. México: Siglo XXI- CIIH, UNAM, reedición 2001. 